\title{
Latest ATLAS measurements of inclusive and differential top-quark pair production cross-sections
}

\author{
Liza Mijović*广 \\ SUPA - School of Physics and Astronomy, University of Edinburgh, United Kingdom \\ E-mail: liza.mijovicecern.ch
}

\begin{abstract}
Latest ATLAS measurements of inclusive and differential top-quark pair production crosssections are reviewed. All the measurements use LHC data taken at the centre-of-mass energy of $13 \mathrm{TeV}$. Prospects for increasing the measurement precision and the potential of the measurements for generator tuning are discussed.
\end{abstract}

7th Annual Conference on Large Hadron Collider Physics - LHCP2019

20-25 May, 2019

Puebla, Mexico

* Speaker.

$\dagger^{\dagger}$ on behalf of the ATLAS Collaboration 


\section{Introduction}

The Large Hadron Collider (LHC) has delivered over 100 million top-quark pair production $(t \bar{t})$ events to the LHC experiments. The precise inclusive and differential $t \bar{t}$ cross-section $\left(\sigma_{t \bar{t}}\right)$ measurements with these events provide insights in two regimes:

- In the case of observables for which high-precision theory predictions are available, the measurements provide precision tests of the Standard Model (SM) at high energy scales. These are used to look for small deviations indicating the presence of Beyond the Standard Model (BSM) physics.

- When the measured quantities are hard to calculate or model precisely, the measurements enable setting constraints on the model parameters. This reduces the $t \bar{t}$ modelling uncertainties. More precise modelling benefits subsequent measurements or searches with large backgrounds from the $t \bar{t}$ production.

This report discusses both regimes; the precision SM tests and measurements constraining parameters of the generator models. The measurements use the data taken by the ATLAS experiment [1] at the centre-of-mass energy of $13 \mathrm{TeV}$ with integrated luminosities between $3.2 \mathrm{fb}^{-1}$ and $36.1 \mathrm{fb}^{-1}$. The data is part of the $139 \mathrm{fb}^{-1}$ LHC Run-2 data-set which was collected in 2015-2018.

\section{Inclusive $t \bar{t}$ Cross-Section Measurements}

The most precise inclusive $t \bar{t}$ cross-section was measured in events containing opposite-signed $e \mu$ events and at least one $b$-tagged jet [2]. In this channel the backgrounds from processes other than $t \bar{t}$ are only about $10 \%$, which keeps the background-related uncertainties low. The analysis approach which enables the high precision extends the simple cut-and-count strategy. In the cutand-count approach the cross-section $\sigma$ is extracted from the number of events passing the selection cuts $N$ :

$$
N=N^{\mathrm{sig}}+N^{\mathrm{bkg}}=\mathscr{L}_{\text {int }} \sigma \varepsilon+N^{\mathrm{bkg}}
$$

where $\mathscr{L}_{\text {int }}$ is the integrated luminosity, $N^{\text {sig }}$ and $N^{\text {bkg }}$ the number of the signal and background events and $\varepsilon$ the efficiency of the signal events to pass the selection requirements. In case $\varepsilon$ is extracted from the simulated events, it is affected by modelling uncertainties of the underlying process and uncertainties of simulating the detector response. These effects are largely circumvented in the inclusive $\sigma_{t \bar{t}}$ measurement by simultaneously extracting the $\sigma$ and the efficiency from the data. The number of observed events with exactly one $b$-tagged jet $\left(N_{1}\right)$ and exactly two $b$-tagged jets $\left(N_{2}\right)$ is related to the $\sigma_{t \bar{t}}$ and efficiency as:

$$
\begin{aligned}
& N_{1}=N_{1}^{\mathrm{sig}}+N_{1}^{\mathrm{bkg}}=\mathscr{L}_{\mathrm{int}} \sigma_{t \bar{t}} \varepsilon_{e \mu} 2 \varepsilon_{b}\left(1-C_{b} \varepsilon_{b}\right)+N_{1}^{\mathrm{bkg}} \\
& N_{2}=N_{2}^{\mathrm{sig}}+N_{2}^{\mathrm{bkg}}=\mathscr{L}_{\mathrm{int}} \sigma_{t \bar{t}} \varepsilon_{e \mu} C_{b} \varepsilon_{b}^{2}+N_{2}^{\mathrm{bkg}},
\end{aligned}
$$

where $N_{1}^{\mathrm{sig}}$ and $N_{1}^{\mathrm{bkg}}\left(N_{2}^{\mathrm{sig}}\right.$ and $\left.N_{2}^{\mathrm{bkg}}\right)$ are the number of signal and background events with exactly one (two) $b$-tagged jets, $\varepsilon_{e \mu}$ is the efficiency of the $e \mu$ pair to pass the analysis requirements such 
as $|\eta|<2.5$ requirement ${ }^{1}$ and $\varepsilon_{b}$ is the efficiency of jets associated with $b$-quarks to pass selection and $b$-tagging identifications requirements. The $C_{b}$ is the $b$-tagging correlation coefficient evaluated as $C_{b}=\varepsilon_{b b} / \varepsilon_{b}^{2}$ where $\varepsilon_{b b}$ is the efficiency of two $b$-jets passing the requirements simultaneously. The $C_{b}$ and $\varepsilon_{e \mu}$ are extracted from the simulated signal events. The two equations of Eq.(2.2) enable two quantities to be extracted from the data simultaneously: the $\sigma_{t \bar{t}}$ and the $\varepsilon_{b}$. The in-situ measurement of the $\varepsilon_{b}$ is key to the high precision, since it makes the analysis largely unaffected by the uncertainties associated with jet energy scale, $b$-tagging efficiency and uncertainties related to the generator modelling of jet production. The measured cross-section value is:

$$
\sigma_{t \bar{t}}=818 \pm 8 \text { (stat) } \pm 27 \text { (syst) } \pm 19 \text { (lumi) } \pm 12 \text { (beam) pb }
$$

where the four uncertainties arise from data statistics, experimental and theoretical systematic effects, the integrated luminosity and the LHC beam energy. The measurement is consistent with the theory prediction obtained at next-to-next-to-leading-order accuracy, including the resummation of next-to-next-to-leading logarithmic soft gluon terms [3]:

$$
\sigma_{t \bar{t}}=832 \pm 35\left(\mathrm{PDF} / \alpha_{\mathrm{S}}\right)_{-29}^{+20}(\text { scale }) \mathrm{pb}
$$

where the uncertainties are from the parton distribution function (PDF) and the choice of the renormalization and factorization scale. The relative uncertainty of the prediction is ${ }_{-5.5 \%}^{+4.8 \%}$, slightly higher compared to the measurement uncertainty of only $4.4 \%$ !

The measurement in Eq. (2.3) was obtained with $3.2 \mathrm{fb}^{-1}$ of data and is dominated by the systematic uncertainties. The systematic uncertainty is dominated by the effects of the parton shower and hadronization on the $\varepsilon_{e \mu}$ acceptance. This uncertainty is reducible by using experimental measurements to constrain the model parameters or in the so-called fiducial measurements done only within the experimental acceptance of the leptons. The uncertainty due to the integrated luminosity has also been substantially reduced for the full LHC Run-2 data-set [4]. Further, the LHC measurements have strong potential to constrain the PDF uncertainties thus the uncertainty on the theory prediction in Eq. (2.4) is expected to decrease. In the future the reduced $\sigma_{t \bar{t}}$ measurement and theory prediction uncertainties will enable even higher precision tests of the SM.

\section{Differential $t \bar{t}$ Cross-Section Measurements}

The differential $t \bar{t}$ cross-section measurements can be used in BSM searches, for example looking for new high-mass particles. The measurements have also had a large impact on improving the generator modelling [5] and constraining PDF uncertainties [6, 7]. The large amount of data collected during Run-2 enables the measurements to probe hard-process scales up to $O(\mathrm{TeV})$. In such events the incoming partons may carry up to one third of the proton's energy and the collision products are highly collimated due to the large Lorentz boost. The boosted hadronicallydecaying top quark can be reconstructed as a single large-radius (large- $R$ ) jet from the 3-body decay $(t \rightarrow b W, W \rightarrow q \bar{q})$. This Section reviews recent boosted $t \bar{t}$ differential cross-section measurements. The large- $R$ jets are constructed using the anti- $k_{t}$ clustering algorithm [8] and $R=1.0$.

\footnotetext{
${ }^{1}$ ATLAS uses a right-handed coordinate system with its origin at the nominal interaction point (IP) in the centre of the detector and the $z$-axis along the beam pipe. The $x$-axis points from the IP to the centre of the LHC ring, and the $y$-axis points upward. Cylindrical coordinates $(r, \phi)$ are used in the transverse plane, $\phi$ being the azimuthal angle around the $z$-axis. The pseudorapidity is defined in terms of the polar angle $\theta$ as $\eta=-\ln \tan (\theta / 2)$.
} 


\subsection{Boosted Differential Cross-Section in Hadronic Decay Channel}

In the boosted events in which both the $t$ and $\bar{t}$ decay hadronically, the $t \bar{t}$ system can be constructed from two large- $R$ jets. The jets can be used to measure the boosted differential crosssections as a function of the $t \bar{t}$ system kinematic properties. Such measurements were performed with $36.1 \mathrm{fb}^{-1}$ of data taken by ATLAS, requesting events with two large-radius jets in the final state, one with transverse momentum $p_{\mathrm{T}}>500 \mathrm{GeV}$ and a second with $p_{\mathrm{T}}>350 \mathrm{GeV}$ [9]. The top quark candidates were separated from the dominant multijet background using jet substructure information and association with a $b$-tagged jet. The unfolded spectra of the transverse momentum $\left(p_{\mathrm{T}}^{t \bar{t}}\right)$ and invariant mass $\left(m^{t \bar{t}}\right)$ of the $t \bar{t}$ system are shown in Figures 1a and $1 \mathrm{~b}$.

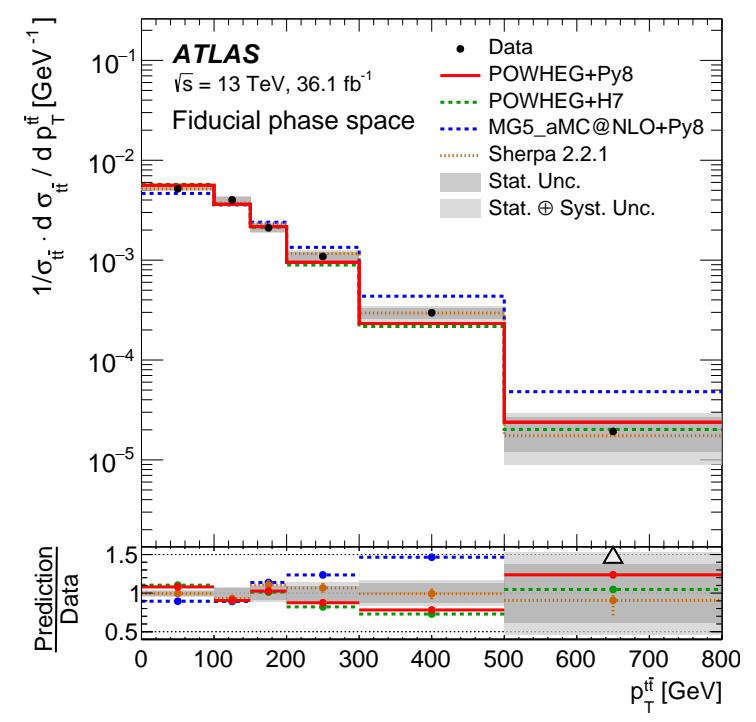

(a)

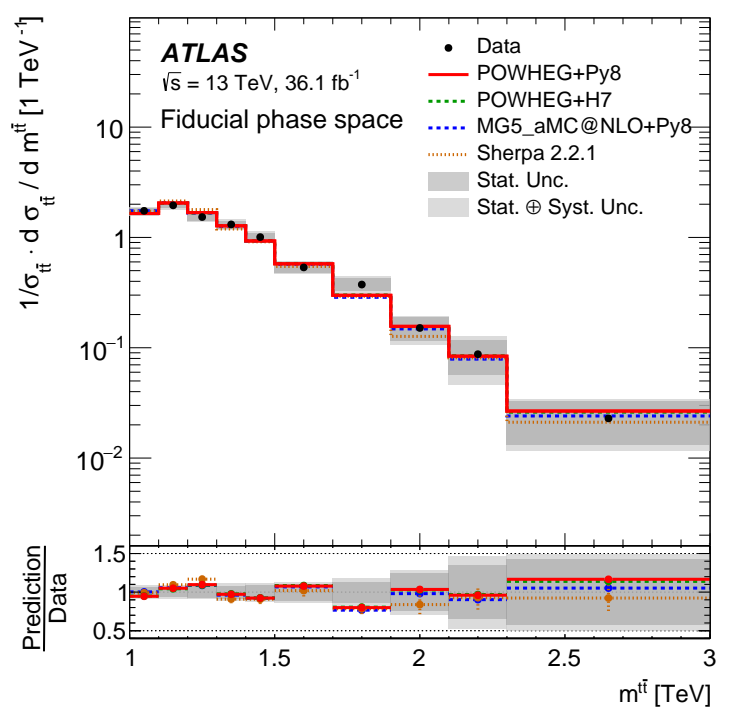

(b)

Figure 1: Normalized differential cross-sections in boosted $t \bar{t}$ events as a function of a) $p_{\mathrm{T}}{ }^{t \bar{t}}$ and b) $m^{t \bar{t}}$. The measurements are corrected for detector effects to particle level. The gray bands indicate the statistical and total uncertainties in the data in each bin. Source: [9].

The $p_{\mathrm{T}}{ }^{t \bar{t}}$ is found to be mis-modelled by one of the main generators used by ATLAS. The $m^{t \bar{t}}$ spectrum extends up to $3 \mathrm{TeV}$ and is found to be in good agreement with the generator model predictions. Besides the spectra, the inclusive cross-section within the detector acceptance is measured to be $\sigma_{t \bar{t}}^{\text {boosted }}=292 \pm 7$ (stat) \pm 76 (syst) fb. The measured $\sigma_{t \bar{t}}^{\text {boosted }}$ is found to be systematically lower (by about one standard deviation) compared to the theory predictions obtained from the generator models. Since only about 3500 events pass the event selection criteria, the analysis is limited by statistical uncertainty at high $p_{\mathrm{T}}{ }^{t \bar{t}}$ and $m^{t \bar{t}}$ and its sensitivity will improve with the full Run-2 data-set.

\subsection{Boosted Jet Substructure in $t \bar{t}$ Events}

Measurements such as the boosted $\sigma_{t \bar{t}}$ measurement of Section 3.1 rely on the large- $R$ jet substructure to identify jets from hadronically-decaying $W$ bosons and top quarks. In order to effectively use these variables it is crucial that they are well modelled by the generators. The 
measurement in Ref. [10] checks whether this is the case. The analysis measures observables sensitive to the pronged substructure such as $N$-subjetiness [11] and energy correlation functions, unfolds the spectra to the particle level and compares them to several modern generators.

The $N$-subjetiness describes to what degree a substructure of a given jet is compatible with being composed of $N$ or fewer subjets. It is calculated by defining $N$ subjet axes within the jet using the exclusive $k_{t}$ algorithm where the subjet reconstruction continues until a desired number of jets are found. The 0-, 1-, 2-,and 3-subjettiness are defined as:

$$
\begin{array}{lc}
N=0: & \tau_{0}=\sum p_{\mathrm{T}_{i}} R \\
N=1: & \tau_{1}=\frac{1}{\tau_{0}} \sum p_{\mathrm{T}_{i}} \Delta R_{a 1, i} \\
N=2: & \tau_{2}=\frac{1}{\tau_{0}} \sum p_{\mathrm{T}_{i}} \min \left(\Delta R_{a 1, i}, \Delta R_{a 2, i}\right) \\
N=3: \tau_{3}=\frac{1}{\tau_{0}} \sum p_{\mathrm{T}_{i}} \min \left(\Delta R_{a 1, i}, \Delta R_{a 2, i}, \Delta R_{a 3, i}\right)
\end{array}
$$

where the sum runs over all jet constituents and $\Delta R_{a n, i}$ is the angular distance between constituent $i$ and the axis of the $n^{\text {th }}$ subjet. The ratios $\tau_{21}=\tau_{2} / \tau_{1}$ and $\tau_{32}=\tau_{3} / \tau_{2}$ are useful to identify the jets which are likely to come from the 2- and 3-body decays respectively, such as hadronic decays of the $W$ boson and the top quark. These observables are shown in Figure 2a and Figure 2b. In Figure 2a the jets are enriched with jets from hadronically-decaying $W$ bosons by requesting $60 \mathrm{GeV}<$ jet mass $<100 \mathrm{GeV}, 1<\Delta R$ (large- $R$ jet, $b$-tagged jet $)<1.8$. In Figure $2 \mathrm{~b}$ the jets are enriched in hadronically-decaying top jets by requiring: jet mass $>140 \mathrm{GeV}, \Delta R$ (large- $R$ jet, $b$ tagged jet) $<1$.

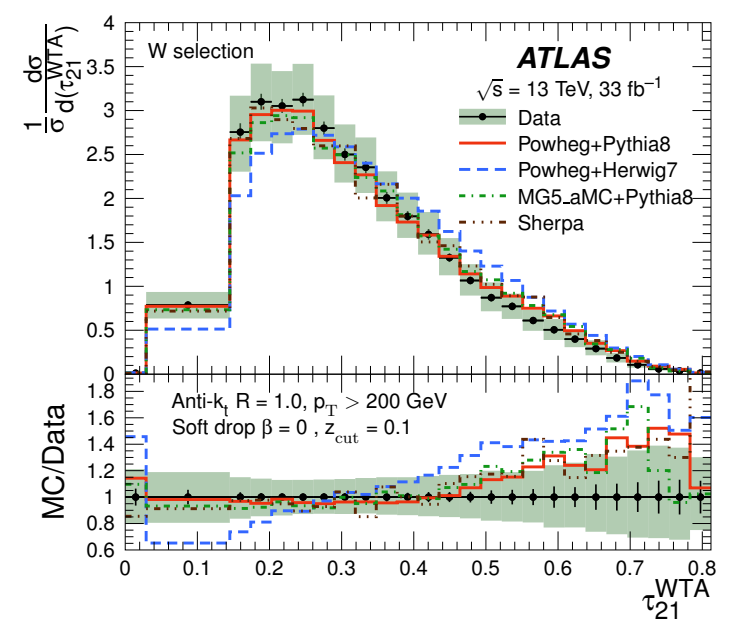

(a)

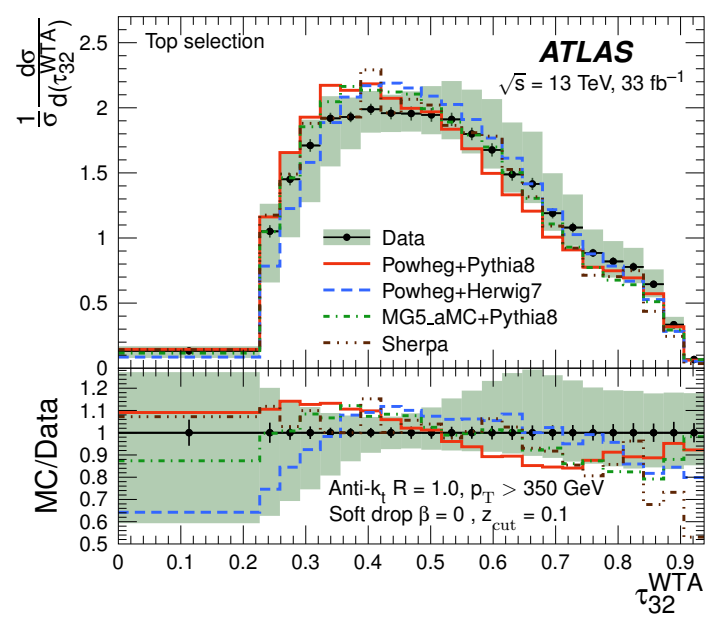

(b)

Figure 2: The distributions of a) $\tau_{21}$ and b) $\tau_{32}$ variables compared to various generator predictions. The subscript WTA indicates that Winner Takes All axis choice was used when calculating the observables. The shaded bands represent the total uncertainty. Source: [10].

The generators were found to describe the data well with the exception of the HERWIG7 generator with the MMHT2014 H7UE tune [12]. This tune significantly mis-models the $N$-subjetiness ratio $\tau_{21}$ as well as several other substructure observables. 


\section{Summary \& Outlook}

The inclusive $t \bar{t}$ cross-section measurements by the ATLAS collaboration are consistent with the SM predictions and have reached relative uncertainties comparable to the state-of-the-art theory predictions $(4 \%-5 \%)$. With the full Run-2 data-set both measurement and theory prediction uncertainties are expected to decrease, enabling higher precision tests of the SM.

The large LHC data-set enables measurements at $O(\mathrm{TeV})$ scales, in which the decay products from a hadronically-decaying top quark or $W$ boson are boosted and reconstructed as large- $R$ jets. The ATLAS differential boosted cross-section measurements provide valuable inputs to the generator tuning. They are statistically limited and will gain sensitivity once the full Run-2 data-set has been analysed.

\section{References}

[1] ATLAS Collaboration, The ATLAS Experiment at the CERN Large Hadron Collider, JINST 3 (2008) S08003.

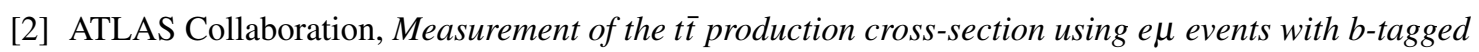
jets in pp collisions at $\sqrt{s}=13 \mathrm{TeV}$ with the ATLAS detector, Phys. Lett. B761 (2016) 136 arXiv:1606.02699 [hep-ex].

[3] M. Czakon, P. Fiedler and A. Mitov, Total Top-Quark Pair-Production Cross Section at Hadron Colliders Through $O\left(\alpha_{S}^{4}\right)$, Phys. Rev. Lett. 110 (2013) 252004 arXiv: 1303.6254 [hep-ph].

[4] ATLAS Collaboration, Luminosity determination in pp collisions at $\sqrt{s}=13 \mathrm{TeV}$ using the ATLAS detector at the LHC, ATLAS-CONF-2019-021

[https://cdsweb.cern.ch/record/2677054] (2019).

[5] ATLAS Collaboration, Improvements in $t \bar{t}$ modelling using NLO+PS Monte Carlo generators for Run2, ATL-PHYS-PUB-2018-009 [https : / / cds. cern. ch/record/2630327] (2019).

[6] ATLAS Collaboration, Determination of the parton distribution functions of the proton from ATLAS measurements of differential $W$ and $Z / \gamma^{*}$ and $t \bar{t}$ cross sections, ATL-PHYS-PUB-2018-009 [https://cdsweb.cern.ch/record/2633819] (2019).

[7] M. Czakon, N. P. Hartland, A. Mitov, E. R. Nocera and J. Rojo, Pinning down the large-x gluon with NNLO top-quark pair differential distributions, JHEP 04 (2017) 044 arXiv : 1611.08609 [hep-ph].

[8] M. Cacciari, G. P. Salam and G. Soyez, The anti-kt jet clustering algorithm, JHEP 04 (2008) 063 arXiv:0802.1189 [hep-ph].

[9] ATLAS Collaboration, Measurements of $t \bar{t}$ differential cross-sections of highly boosted top quarks decaying to all-hadronic final states in pp collisions at $\sqrt{s}=13 \mathrm{TeV}$ using the ATLAS detector, Phys. Rev. D98 (2018) 012003 arXiv: 1801.02052 [hep-ex].

[10] ATLAS Collaboration, Measurement of jet-substructure observables in top quark, W boson and light jet production in proton-proton collisions at $\sqrt{s}=13 \mathrm{TeV}$ with the ATLAS detector, JHEP 08 (2019) 033 arXiv: 1903.02942 [hep-ex].

[11] J. Thaler and K. Van Tilburg, Identifying Boosted Objects with N-subjettiness, JHEP 03 (2011) 015 arXiv:1011.2268 [hep-ph].

[12] J. Bellm et al., Herwig 7.0/Herwig++ 3.0 release note, Eur. Phys. J. C76 (2016) 196 arXiv:1512.01178 [hep-ph]. 